\title{
Propiedades psicométricas de la versión internacional de la Escala de Afecto Positivo y Negativo-forma corta (I- Spanas SF) en estudiantes universitarios
}

Rafael Gargurevich Universidad de Lima, Perú

Recibido: 15 de julio del 2010 / Aprobado: 11 de agosto del 2010

El objetivo de la presente investigación fue estudiar las propiedades psicométricas de la versión internacional de la Escala de Afecto Positivo Negativo-forma corta -traducción al español de International Positive and Negative Affect Schedule-Short Form (I-Spanas-SF) - en estudiantes universitarios de Lima. El I-Spanas-SF utiliza solo 10 de los 20 items originales del Spanas. Sus propiedades psicométricas fueron evaluadas en una muestra amplia de personas provenientes de 52 países. Los resultados de los análisis factoriales exploratorio y confirmatorio demostraron que la escala I-Spanas-SF está conformada por dos subescalas, las que coinciden con la estructura bifactorial planteada por el Spanas.

afecto positivo / afecto negativo / Spanas / análisis factorial confirmatorio

Psychometric properties of the International Short Version of the Positive and Negative Affect Schedule (I-Spanas-SF) in university students

The present research studies the psychometric properties of the Spanish short version of the International Positive and Negative Affect Schedule (I-Spanas-SF) applied to university students in Lima. The Panas (Positive and Negative Affect Schedule) has 20 items and 2 sub-scales (10 for each one) that measures positive and negative affects. The I-Panas-SF only uses 10 of the original items of the Spanas. Psychometric properties were previously evaluated on a large sample of subjects from 52 countries. Results of the exploratory and confirmatory factorial analyses show that the I-Spanas-SF is composed of two sub-scales, which coincide with the bi-factorial structure first designed for the Spanas.

positive affect / negative affect / Spanas / confirmatory factorial analysis

Correo electrónico: rgargure@ulima.edu.pe. 


\section{El afecto y el Panas}

Las experiencias afectivas son parte de la vida diaria de las personas y representan uno de los campos de estudio más importantes en psicología.

El afecto ha sido descrito como un elemento disposicional (Zajonc, 1980; Sandín et al., 1999; Thompson, 2007) en el que existen básicamente dos grandes grupos de experiencias: las de afecto positivo y las de afecto negativo (Watson \& Tellegan, 1985; Watson \& Clark, 1994). El afecto positivo se refleja en las experiencias gratificantes, que producen bienestar y se siente entusiasmo y alegría, mientras que el afecto negativo se refleja en situaciones en que surgen el disgusto, la ira o el miedo (Watson \& Tellegan, 1985; Sandín et al., 1999; Ostir et al., 2005). Además, los afectos negativo y positivo correlacionan positivamente con otros rasgos de la personalidad. El afecto negativo correlaciona positivamente con neuroticismo y el afecto positivo hace lo propio con rasgos como la extroversión, la apertura o la agradabilidad (Watson \& Clark, 1994).

Estos estados afectivos permiten realizar una rápida discriminación de aquello que puede ser considerado como "bueno" o "malo" (Watson \& Clark, 1994; Gross \& Thompson, 2007) y ser visto como activador de las respuestas emocionales tanto positivas como negativas (Crawford \& Henry, 2004). Si bien los afectos negativo y positivo han sido concebidos como ortogonales (Watson, Clark \& Tellegen, 1988), diversos estudios empíri$\cos$ factoriales han comprobado que esto no es necesariamente así, es decir, la presencia de un estado afectivo no elimina por completo la presencia del otro (DePaoli \& Sweeney, 2000; Crawford \& Henry, 2004).

Uno de los instrumentos más populares para investigar los estados afectivos positivo y negativo ha sido el Panas (Positive and Negative Affect Schedule) (Watson, Clark \& Tellegen, 1988), debido en parte a sus excelentes propiedades psicométricas. El Panas ha sido adaptado y estudiado en diversos idiomas, como el español (Joiner, Sandin, Chorst, Lostao \& Marquina, 1997; Sandín et al., 1999), el holandés (Petters et al., 1996), el alemán (Krohne, Egloff, Kohlmann \& Tausch, 1996), el ruso (Balatsky \& Diener, 1993), el sueco (Hilleras, Jorm, Herlitz \& Winblad, 1998), el turco (Gencoz, 2000), el italiano (Terracciano, McCrae \& Costa, 2003), entre otros, y en todos estos estudios el Panas ha demostrado ser un instrumento válido y confiable.

En el Perú, la evidencia empírica de la aplicación del Panas es en realidad escasa, no obstante su popularidad en el ámbito internacional. Las propiedades psicométricas de la prueba en el Perú ha sido estudiada por primera vez por Grimaldo (2003), quien ha reportado la validez y confiabilidad del Spanas, versión en español del Panas, 
en estudiantes de cuarto y quinto de secundaria $(\mathrm{N}=589)$. Luego de realizar un análisis de los componentes principales, Grimaldo (2003) reportó una estructura factorial de acuerdo con lo esperado para la prueba compuesta por dos factores: uno con relación al afecto positivo y otro con relación al afecto negativo. Recientemente, Matos (2009) aplicó el Panas en una muestra de 580 estudiantes universitarios como parte de un estudio acerca de la relación entre la motivación de los estudiantes, las estrategias de aprendizaje, el afecto y el rendimiento académico. Matos (2009) declaró que al estudiar el Panas mediante el análisis factorial confirmatorio (AFC) encontró una estructura bifactorial, que coincidía con las subescalas de afecto positivo y negativo de la prueba, las que además resultaron ser confiables.

\section{El PANAS de Forma CORTA}

Otra de las razones por las que el Panas se ha convertido en un instrumento tan popular es la rapidez de su aplicación. Consta de una lista de 20 palabras, 10 de las cuales identifican el afecto positivo (por ejemplo, "Activo") y 10 el afecto negativo (por ejemplo, "Irritable"), y el participante debe decidir en un rango del 1 ("Levemente") al 5 ("En extremo") si el ítem representa alguno de sus estados afectivos.

A pesar de la rapidez en su aplicación, algunas investigaciones utilizan un gran número de cuestionarios o tienen un tiempo de recolección de información muy limitado, por lo que se necesitan instrumentos aun más breves. Además, el desarrollo de una forma corta del Panas no solo se relaciona con la funcionalidad de una prueba corta. Diversos estudios acerca de la estructura factorial del Panas han reportado la necesidad de calcular correlaciones entre los errores de ciertas parejas de ítems que pertenecen a una misma subescala para lograr buenos índices de ajuste. De esta manera quedaría en evidencia la existencia de una redundancia en ciertos ítems de la prueba (Thompson, 2007).

Así, Kercher (1992) desarrolló una forma corta del Panas; sin embargo, esta versión presentó algunas dificultades con relación a la validez de constructo de la escala. Al seleccionar los ítems Kercher usó los que tenían las cargas factoriales más altas en el estudio de validación original de la escala (Watson et al., 1988) y no utilizó análisis factoriales confirmatorios para la selección de ítems, lo que originó una alta covariación entre los ítems seleccionados y un reporte excesivamente alto de la confiabilidad de la prueba (Thompson, 2007; Kercher, 1992).

Por esta razón, se desarrolló una nueva forma corta del Panas, llamada I-Panas- SF (International-Panas-Short Form) (Thompson, 2007). Al igual que la versión del Panas de Kercher (1992) esta consta de 10 ítems: cinco evalúan 
el afecto positivo y los otros cinco el afecto negativo (Thompson, 2007).

A diferencia de la versión anterior, el I-Panas-SF fue desarrollado sobre la base de diversos análisis factoriales confirmatorios en los que se aislaron los ítems del Panas con las mayores cargas factoriales. Este procedimiento aseguró la validez de constructo de la escala, ya que se buscó reproducir la estructura bifactorial del Panas. Esta estructura fue comprobada en cuatro estudios diferentes, en los que se evaluaron a alrededor de 2.000 personas pertenecientes a 52 países diferentes y en donde se encontraron cargas factoriales desde .61 a .83 para el afecto positivo y de .63 a .81 para el afecto negativo (Thompson, 2007).

Los índices de consistencia interna del I-Panas-SF alcanzaron valores de .73 a .78 para el afecto positivo y de .72 a .76 para el afecto negativo, lo que es ligeramente menor a la consistencia interna alcanzada por el Panas original (.82 para el afecto positivo y .85 para el afecto negativo) (Thompson, 2007).

La correlación entre las escalas del I-Panas-SF y el Panas fue estudiada en una de las muestras y se reportó un índice $.92(p<.01)$ en caso del afecto positivo y $.95(p<.01)$ en el caso del afecto negativo. La correlación entre las dos escalas del I-Panas-SF negativa fue negativa (entre $r=-.32$ y $r=-.29$, $p<.01)$. Las medias del I-Panas-SF fueron $19.15(D E=2.77)$ y $12.73(D E$
= 3.01) para el afecto positivo y el afecto negativo, respectivamente.

Si bien el Panas ha sido aplicado en diversas investigaciones en el Perú, el I-Panas-SF aún no ha sido utilizado en el país; por lo que el objetivo de la presente investigación es presentar los resultados de las propiedades psicométricas del I-Panas-SF por primera vez en el Perú.

\section{Metodología}

\section{Participantes}

La muestra estuvo conformada por 320 alumnos de una universidad privada, cuya es de alrededor de 21 años $(M=$ 20.91, $D E=2.43$ ), de los cuales 164 (52\%) son de sexo masculino y 156 $(48 \%)$ de sexo femenino.

La mayoría de ellos nacieron en Lima $(N=250,78 \%)$ y estudiaron en colegios privados $(N=291,91 \%)$, mientras que otros nacieron en provincias $(N=34,11 \%)$ o en el extranjero $(N$ $=13,4 \%)$ y estudiaron en colegios públicos $(N=29,9 \%)$. De la muestra total el $29 \%(N=92)$ declaró que sus madres tenían educación superior, mientras que en el caso de los padres el $61 \%(N=195)$ contaba con educación superior.

\section{Instrumentos (I-Panas-SF y Panas)}

Como se mencionó, el cuestionario IPanas-SF es una forma corta del Panas. El I-Panas-SF consta de 10 ítems (5 de 
afecto negativo y 5 de afecto positivo). Al igual que el Panas, el individuo debe declarar con qué frecuencia experimenta las palabras que se le presentan (las que describen diversos estados emocionales). Debido a que el I-Panas-SF utiliza los mismos ítems que el Panas, se utilizaron los ítems de Spanas (Joiner et al., 1997) utilizados en el Perú por Grimaldo (2003) y Matos (2009).

\section{Procedimientos}

Ambos cuestionarios fueron evaluados al mismo tiempo como parte de una batería de cuestionarios más larga. Después de los consentimientos informados, los participantes respondieron el Panas, y luego del resto de cuestionarios los participantes respondieron el I-Panas-SF.

De esta manera el I-Panas-SF fue respondido alrededor de 45 minutos después de que fuera respondido el Panas. Los cuestionarios fueron aplicados en los horarios regulares de clase.

\section{Plan de análisis de datos}

Para analizar las propiedades psicométricas de los cuestionarios se evaluará la validez y confiabilidad de los resultados de ambas pruebas.

La validez de la escala será evaluada a partir de la validez de constructo (factorial, convergente y divergente). Para analizar la validez de constructo de los instrumentos se estudiarán los componentes principales, en el que se examinarán los autovalores, el gráfico de sedimentación de Cattell y las cargas factoriales de los ítems para determinar cuáles de estos forman parte de cada componente.

A continuación se realizará un Análisis Factorial Confirmatorio (AFC) (Joreskog \& Sörbom, 2001) para estar seguros de la estructura factorial de la escala. Los resultados del AFC se evaluarán mediante la inspección de diversos índices de ajuste:

- La proporción del chi cuadrado (que se calcula dividiendo el chi cuadrado entre los grados de libertad), para lo cual es necesario tener información acerca del chi cuadrado $\left(\chi^{2}\right)$ y los grados de libertad (gl). Si los datos no estuvieran distribuidos de manera normal, se empleará el SatorraBentler chi cuadrado (S-B $\left.\chi^{2}\right)$.

- El RMSEA (Raíz Cuadrada Media de Error de Aproximación).

- El GFI (Índice de Bondad de Ajuste). Cuando los valores de la proporción del chi cuadrado se encuentran alrededor de 3 indican una buena adecuación del modelo (Kline, 1998). Valores cercanos a 0.08 en el RMSEA y valores superiores a 0.90 en el GFI muestran una buena adecuación del modelo ( $\mathrm{Hu}$ \& Bentler, 1999; Kline, 1998).

La validez convergente se evaluará realizando una correlación entre las escalas de afecto positivo y negativo del Panas y del I-Panas-SF. Se espera que 
escalas de afecto positivo y negativo en el I-Panas-SF correlacionen significativamente con las escalas equivalentes del Panas. Para evaluar la validez divergente, la correlación entre las escalas que evalúan constructos teóricos diferentes debería ser negativa o no significativa. En este caso, la escala de afecto negativo del I-Panas-SF debería correlacionar negativamente con la escala de afecto positivo del Panas y en el caso del afecto positivo del I-Panas-SF esta escala debe correlacionar negativamente con la escala de afecto negativo del Panas.

Para analizar la confiabilidad de las escalas se empleará el método de consistencia interna, en el que se incluirá toda la muestra. Se debe revisar la correlación de cada ítem con el total del factor, el cual debe ser igual o mayor a .30. El índice de consistencia interna (alfa de Cronbach) debe ser de al menos .70 para ser considerado aceptable (Aiken, 1996).

\section{Resultados}

La evaluación de la validez de constructo de las pruebas se efectuó en dos partes:

- Se realizaron análisis para verificar la validez factorial de las escalas mediante análisis factoriales exploratorios (AFE) y confirmatorios (AFC).

- Se hicieron análisis correlacionales para establecer la validez convergente y divergente del instrumento.
A continuación se presentan los resultados de los análisis realizados.

Para iniciar la exploración validez de constructo del I-Panas-SF se realizó un análisis factorial exploratorio, por lo que se llevó a cabo un análisis de componentes principales con rotación Varimax, dado que teóricamente ambos constructos (afecto positivo y negativo) son ortogonales (Watson, Clark y Telegen, 1988).

El resultado de estos análisis evidenció que la medida de adecuación de la muestra Kayse-Meyer-Olkin (KMO) fue de .77 , y el test de esfericidad de Bartlett fue altamente significativo $(p<$ .000 ), por lo que se pudo continuar con la interpretación de los resultados del análisis. El resultado de estos análisis mostró que dos componentes explicaban el 49,48\% de la varianza. El primer componente presentó un autovalor de 2.63 el cual explicaba el $26,33 \%$ de la varianza y el segundo componente presentó un autovalor de 2.32 , lo que explicó el 23,15\% adicional de varianza. Sin embargo, esta estructura bifactorial encontrada no logró ser corroborada por el gráfico de sedimentación de Cattell, ya que en este se observa que podrían retenerse más de dos componentes (figura 1). Las cargas factoriales de los ítems de las escalas de afecto positivo y negativo alcanzaron valores entre 0.59 y 0.85 (véase la tabla 1).

Para corroborar la estructura factorial de la escala se realizó un análisis factorial confirmatorio (AFC) utilizan- 
Flgura 1

Gráfico de sedimentación del I-Panas-SF

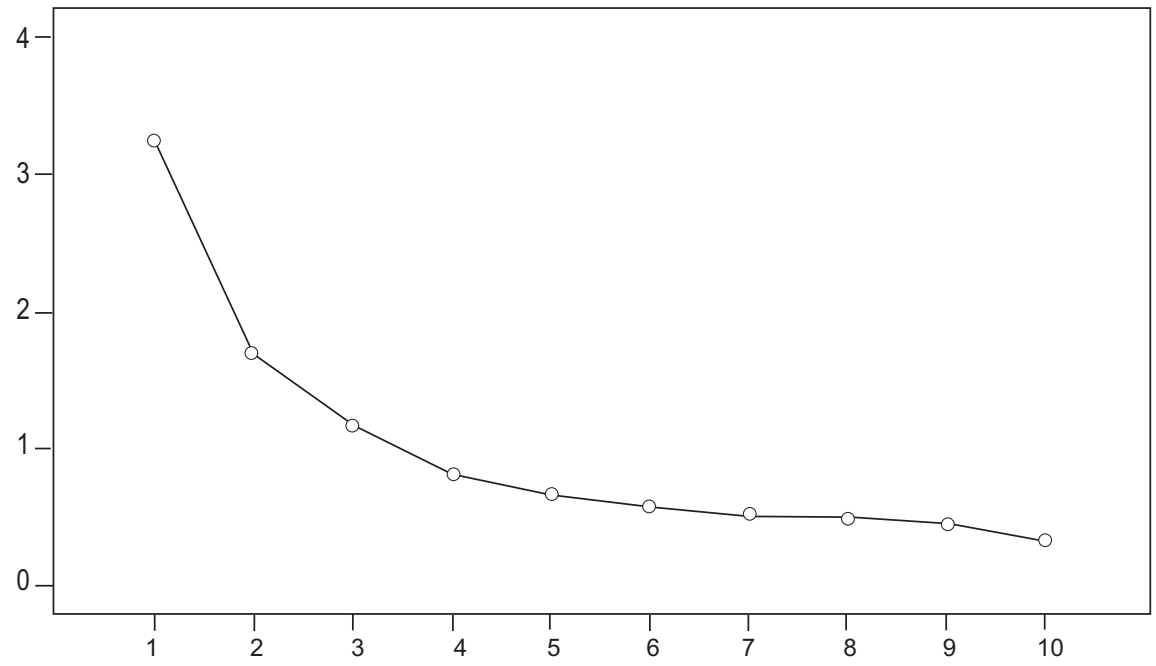

Tabla 1

Cargas factoriales resultantes del análisis de componentes principales con rotación Varimax y del AFC del I-PANAS-SF $(\mathrm{N}=320)$

\begin{tabular}{|c|c|c|c|c|}
\hline \multirow[t]{2}{*}{ Ítems } & \multicolumn{2}{|c|}{ Exploratorio } & \multicolumn{2}{|c|}{ Confirmatorio } \\
\hline & Negativo & Positivo & Negativo & Positivo \\
\hline 1 & .64 & & $.27^{*}$ & \\
\hline 2 & .72 & & $.38^{*}$ & \\
\hline 3 & & .71 & & $.51^{* * *}$ \\
\hline 4 & .64 & & $.69^{\star \star \star}$ & \\
\hline 5 & & .47 & & $.45^{\star * *}$ \\
\hline 6 & .64 & & $.68^{* * *}$ & \\
\hline 7 & & .71 & & $.69^{* * *}$ \\
\hline 8 & & .67 & & $.55^{\star \star *}$ \\
\hline 9 & .68 & & $.83^{\star \star \star}$ & \\
\hline 10 & & .74 & & $.72^{\star \star *}$ \\
\hline
\end{tabular}

Nota. ${ }^{*} p<0.05,{ }^{* *} p<0.01,{ }^{* * *} p<0.001$ 
do el programa LISREL versión 8.5 (Jöreskog \& Sörbom, 2001). En este análisis se propuso reproducir los resultados del análisis factorial exploratorio. Como ya fue mencionado, los índices de bondad de ajuste evaluados en el AFC fueron: el Satorra-Bentler chi cuadrado $\left(\mathrm{S}-\mathrm{B} \chi^{2}\right)$, la razón del chi cuadrado ( $\mathrm{S}-\mathrm{B} \chi^{2} / \mathrm{gl}$ ), el RMSEA y el CFI. Los resultados mostraron índices de ajuste bastante satisfactorios: S$\mathrm{B} \chi^{2} / \mathrm{gl}=3.27\left(\mathrm{~S}-\mathrm{B} \chi^{2}=111.19, \mathrm{gl}=\right.$ 34), $\mathrm{RMSEA}=0.084$, GFI $=0.92$. Las cargas factoriales de los ítems alcanzaron valores entre 0.27 y 0.83 , siendo todas significativas (tabla 1). No se correlacionó ningún error de covarianzas entre los ítems a pesar de que los índices de modificación lo sugerían. La correlación entre las escalas de afecto positivo y negativo fue negativa y significativa $(r=-0.52, p<0.001)$.

La validez convergente y divergente de las escalas fue evaluada a partir de los resultados de las correlaciones entre las variables evaluadas (tabla 2). Los resultados mostraron que el IPanas-SF obtuvo una correlación positiva significativa con la escala de afecto positivo del Panas $(r=.34, p<.001)$. A su vez obtuvo una correlación negativa significativa con la escala de afecto negativo del Panas $(r=-.18, p<$ $.001)$. La escala de afecto negativo del I-Panas-SF correlacionó de forma positiva y significativa con la escala de afecto negativo del Panas $(r=.54, p<$ .001) y además correlacionó negativa- mente con la de afecto positivo del Panas $(r=-.14, p<.05)$.

Luego de hacer los análisis de validez del I-Panas-SF se realizaron los análisis para comprobar la confiabilidad de las escalas. Para analizar la confiabilidad de las escalas obtenidas en los análisis factoriales se empleó el método de consistencia interna.

En el caso del I-Panas-SF el índice de consistencia interna alfa de Cronbach para el afecto negativo fue de .72 y los índices de correlación ítem-test alcanzaron valores de .33 a .60. En el caso del afecto positivo el índice de consistencia interna fue de .72 y los índices de correlación ítemtest alcanzaron valores de .36 a .56 .

Tabla 2

Resultado del análisis de correlación entre las variables del I-Panas-SF y el Spanas

\begin{tabular}{lccc}
\hline Variables & $\mathbf{1}$ & $\mathbf{2}$ & $\mathbf{3}$ \\
\hline 1. I-Panas-SF positivo & 1 & & \\
2. I-Panas-SF negativo & $-.32^{\star * *}$ & 1 & \\
3. Spanas positivo & $.34^{* * *}$ & $-14^{*}$ & 1 \\
4. Spanas negativo & $-.18^{* *}$ & $.54^{* * *} .25^{* * *}$ \\
\hline
\end{tabular}

Nota. ${ }^{*} p<0.05,{ }^{* *} p<0.01,{ }^{* * *} p<0.001$

\section{DISCUSIÓN}

En la presente investigación se estudiaron las propiedades psicométricas del cuestionario I-Panas-SF en español (ISpanas-SF). Específicamente se estudió la validez de constructo (factorial convergente y divergente) y la consistencia interna de la escala. 
Luego de realizar los análisis de validez y confiabilidad se encontró que el ISpanas-SF posee validez de constructo y ambas escalas (afecto positivo y negativo) han demostrado ser confiables. Los resultados del análisis factorial exploratorio fue verificado por el análisis factorial confirmatorio en el que se encontró que la estructura bifactorial propuesta por la prueba era aceptable.

La validez y confiabilidad de la escala en forma corta resulta particularmente importante para la investigación en el Perú, donde a pesar del esfuerzo por llevarla a cabo aún hay poca evidencia de la adaptación de instrumentos psicológicos. Concretamente, este es el primer instrumento que en forma breve logra evaluar las experiencias afectivas.

Sin embargo, aún hay mucho por hacer. Una de las limitaciones de esta investigación está relacionada con los participantes. En esta investigación, los participantes son todos estudiantes universitarios, lo que asegura la comprensión de las instrucciones y de los ítems. Este grupo solo representa a un sector de la población del país, por lo que deben llevarse a cabo un mayor número de estudios antes de sostener que la estructura bifactorial de la prueba puede ser generalizada a todo el país. Este trabajo sirve como un antecedente (evidencia empírica) y como base para continuar investigando con el ISpanas-SF.

\section{REFERENCIAS}

Balatsky, G. \& Diener, E. (1993). Subjective well-being among Russian students. Social Indicators Research, 28, 225-243.

Crawford, J. R. \& Henry, J. D. (2004). The Positive and Negative Affect Schedule (Panas): Construct validity, measurement properties and normative data in a large non-clinical sample. British Journal of Clinical Psychology, 43, 245-265.

DePaoli, L. \& Sweeney, D. C. (2000). Further validation of the Positive and Negative Affect Schedule. Journal of Social Behavior and Personality, 15, 561-568.

Dyck, M. J.; Jolly, J. B. \& Kramer, T. (1994). An evaluation of positive affectivity, negative affectivity, and hyperarousal as markers for assessing between syndrome relationships. Personality and Individual Differences, 17, 637-646.

Gencoz, T. (2000). Positive and Negative Affect Schedule: A study of validity and reliability. Turk Psikoloji Dergisi, 15, 19-28.

Grimaldo, M. P. (2003). Validez y confiabilidad de la Escala de Afecto Negativo y Positivo (Spanas). Cultura, 21, 341-363.

Gross, J. J. \& Thompson, R. A. (2007). Emotion regulation: Conceptual foundations. En J. J. Gross (Ed.). Handbook of emotion regulation 
(pp. 3-24). Nueva York: Guilford Press.

Hilleras, P. K.; Jorm, A. F.; Herlitz, A. \& Winblad, B. (1998). Negative and positive affect among the very old: A survey on a sample age 90 years or older. Research On Aging, 20, 593-610.

Hu, L. \& Bentler, P. M. (1999). Cutoff criteria for fit indexes in covariance structure analysis: Conventional criteria versus new alternatives. Structu ral Equation Modeling, 6, 1-55.

Joiner, T. E.; Sandín, B.; Chorot, P.; Lostao, L. \& Marquina, G. (1997). Development and factor analytic validation of the Spanas among women in Spain: (More) cross-cultural convergence in the structure of mood. Journal of Personality Assessment, 68, 600-615.

Jöreskog, K. \& Sörbom, D. (2001). Lisrel 8: Structural equation modeling with the Simplis command language. Hillsdale, NJ: Laurence Erlbaum.

Kercher, K. (1992). Assessing subjective well-being in the old-old. The Panas as a measure of orthogonal dimensions of positive and negative affect. Research on Aging, 14, 131168.

Kline, R. B. (1998). Principles and practice of structural equation modeling. Nueva York: The Guilford Press.
Krohne, H.; Egloff, B.; Kohlmann, C. \& Tausch, A. (1996). Investigations with a German version of the positive and negative affect schedule (Panas). Diagnostica, 42, 139-156.

Matos, L. \& Lens, W. (2009). Using a $2 \times 2$ achievement goal framework to predict the use of learning strategies, positive affect and academic achievement in a Peruvian sample of university students. Conferencia Anual de la Asociación Americana de Investigación en Educación. San Diego: California.

Matos, L. (2009). Adaptación de dos cuestionarios de motivación: Autorregulación del Aprendizaje y Clima de Aprendizaje. Persona, 12, 167-185.

Ostir, G. V.; Smith, P. M.; Smith, D. \& Ottenbacher, K. J. (2005). Reliability of the Positive and Negative Affect Schedule (Panas) in medical rehabilitation. Clinical Rehabilitation, 19, 767-769.

Peeters, D.; Ponds, R. \& Vermeeren, M. (1996). Affectiviteit en zelfbeoordeling van depressie en angst. Tijdschrift voor Psychiatri, 3, 240250.

Sandín, B.; Chorot, P.; Lostao, L.; Joiner, T. E.; Santed, M. A. \& Valiente, R. M. (1999). Escalas de Afecto Positivo y Negativo: Validación factorial y convergencia transcultural. Psicothema, 11, 37-51. 
Terracciano, A.; McCrae, R. R. \& Costa, P. T. (2003). Factorial and construct validity of the Italian Positive and Negative Affect Schedule (Panas). European Journal of Psychological Assessment, 19, 131-141.

Thompson, E. R. (2007). Development and Validation of an Internationally Reliable Short-Form of the Positive and Negative Affect Schedule (Panas). Journal of Cross-Cultural Psychology, 38, 227-242.

Watson, D. \& Tellegen, A. (1985). Toward a consensual structure of mood. Psychological Bulletin, 98(2), 219-235.
Watson, D.; Clark, L. A. \& Tellegen, A. (1988). Development and validation of brief measures of positive and negative affect: The Panas scales. Journal of Personality and Social Psychology, 54, 1063-1070.

Watson, D. \& Clark, L. A. (1994). The Panas-X. Manual for the Positive and Negative Affect Schedule Expanded Form. Iowa: The University of Iowa.

Zajonc, R. B. (1980). Feeling and thinking. Preferences need no inferences. American Psychologist, 35, 151-175. 


\section{Apéndice I \\ I-Spanas-SF}

Nombre:

Edad:

A continuación le presentamos varias palabras que describen diferentes sentimientos y emociones. Lea cada una de ellas y marque con un aspa con cuanta frecuencia experimenta usted estas emociones o sentimientos. Por ejemplo, marque 1 si "Nunca" experimenta un sentimiento o emoción, y marque 5 si lo hace "Siempre".

$\begin{array}{ccccc}1 & 2 & 3 & 4 & 5 \\ \text { Nunca } & & & & \text { Siempre }\end{array}$

1. Molesto(a)

12

3

4

5

2. Hostil

1

2

3

$4 \quad 5$

3. Alerta

1

2

3

$4 \quad 5$

4. Avergonzado(a)

1

2

3

$4 \quad 5$

5. Inspirado (a)

2

3

$4 \quad 5$

6. Nervioso(a)

2

3

$4 \quad 5$

7. Decidido(a)

2

3

$4 \quad 5$

8. Atento(a)

2

3

$4 \quad 5$

9. Temeroso(a)

2

3

$4 \quad 5$

10. Activo(a)

1

2

3

4

5 